\title{
Prevalence of postpartum depression and associated factors among women in Mbarara and Rwampara districts of south-western Uganda
}

Catherine Atuhaire* ${ }^{*}$, Godfrey Zari Rukundo, Grace Nambozi, Joseph Ngonzi, Daniel Atwine, Samuel Nambile Cumber and Laura Brennaman

\begin{abstract}
Background: Postpartum depression (PPD) is a significant cause of maternal morbidity and has severe consequences on the well-being of mothers, new-borns, families, and communities. PPD reduces the mother's response to the child's needs. In severe cases, mothers suffering from PPD are prone to postpartum psychosis, commit suicide and, in rare cases, infanticide. We aimed to determine the prevalence and understand the factors associated with PPD among mothers in southwestern Uganda.

Methods: This was a cross-sectional study between November 2019 and June 2020 among 292 mothers, 6 to 8 weeks' postpartum. Mothers were selected from three health facilities in southwestern Uganda and enrolled using stratified consecutive sampling. Postpartum depression was clinically diagnosed using the Diagnostic and Statistical Manual of Mental Disorders V. The factors associated with PPD were assessed by using a structured interviewer administered questionnaire. The factors were analyzed using bivariate chi square analyses and multivariate logistic regression.

Results: Overall prevalence of PPD was $27.1 \%$ (95\% Cl: $22.2-32.5)$. This did not vary by the number of previous births or mode of birth. Five factors associated with PPD were low perceived social support, HIV positive status, rural residence, obstetrical complications and the baby crying excessively.

Conclusion and recommendations: Prevalence of PPD in Mbarara and Rwampara districts is higher than what has previously been reported in Uganda indicating an urgent need to identify pregnant women who are at increased risk of PPD to mitigate their risk or implement therapies to manage the condition. Midwives who attend to these mothers need to be empowered with available methods of mitigating prevalence and consequences of PPD. Women who are HIV positive, residing in rural settings, whose babies cry excessively, having low social support systems and who have birth complications may be a particularly important focus for Ugandan intervention strategies to prevent and reduce the prevalence of PPD.
\end{abstract}

Keywords: Postpartum depression, Prevalence, Associated factors, Uganda

*Correspondence: catuhaire@must.ac.ug

Faculty of Medicine, Department of Nursing, Mbarara University

of Science and Technology (MUST), Mbarara, Uganda

\section{Background}

Postpartum depression (PPD) is a significant cause of maternal morbidity and mortality [1-3]. It also has severe consequences on the physical and social well-being of 
mothers, new-born infants, families and communities. PPD reduces the mother's response to the child's needs and in severe cases, mothers suffering from PPD are prone to postpartum psychosis, suicide and rarely infanticide [4-6]. However, research on PPD has been given minimal attention in low-income countries. Postpartum depression is a non-psychotic disabling mood disorder occurring in the first 6 weeks following childbirth $[7,8]$. During the postpartum period, the woman is expected to recover from the stress of pregnancy, birth and physiological adaptation after childbirth. PPD is characterized by severe mood swings, loss of interest, sleep disturbances, feelings of sadness, excessive crying, fatigability, appetite loss, coping stress with daily activities and thoughts of self-harm or harm to the baby [9]. If risks for PPD are detected early, PPD is preventable and treatable using less risky non-pharmacological methods [10]. Globally, it is estimated that 10 to $15 \%$ of women suffer from PPD [11, 12]. High-income countries have a lower prevalence of PPD compared to low-income countries.

In low-income countries such as Uganda, determining the actual prevalence of postpartum depression remains problematic due to limited screening, diagnosis and under-reporting by postpartum care providers. Although studies on PPD in Africa are limited, available studies report varying prevalence from 6.9 to $39.6 \%$ [12-14]. Most of these studies used screening tools to identify PPD rather than reliable diagnostic criteria [15].

In Uganda, there is paucity of data regarding the prevalence of PPD. A PPD prevalence of $6.1 \%$ was reported in a peri-urban setting and in a study that utilized the Self Reporting Questionnaire (SRQ-20) tool [16]. Another study using the Edinburgh Postnatal Depression Scale (EPDS) reported a prevalence of $43 \%$ in rural Uganda [17]. There is no documented Ugandan study that screened and clinically confirmed PPD.

PPD is associated with socio-demographic, psychosocial, obstetric factors, and influenced by culture in low income countries $[13,18]$. This paper is a step towards understanding factors associated with PPD in the cultural context of Uganda. Determining the prevalence and understanding factors associated with PPD is fundamental to inform policy makers and prepare health care workers (HCW) to implement PPD assessment to conform with Ugandan Clinical Guidelines [19]. Failure to assess and manage PPD has adverse implications towards Uganda's efforts to meet the Sustainable Development Goals (SDG) and the national development targets. Failure to do so has poor mental health effects on the productivity of affected women and their households [19]. Preventing the disorder and promoting mental health has positive implications as compared to treatment of PPD. This study sought to determine prevalence of PPD and factors associated with PPD in Mbarara and Rwampara districts, southwestern Uganda.

\section{Methods \\ Study design}

This paper presents a multi-facility, cross-sectional study that gathered data from mothers during their 6 to 8 weeks' postpartum periods in Mbarara and Rwampara districts, southwestern Uganda.

\section{Study setting}

This study was conducted in Mbarara Regional Referral Hospital (MRRH), Bwizibwera Health Centre IV (BHCIV) and Kinoni Health Centre IV (KHC-IV), approximately $270 \mathrm{~km}$ from Kampala, the capital city of Uganda. Mbarara Regional Referral Hospital (MRRH) is in the urban setting (Mbarara city) and the two HC-IVs, KHCIV in Rwampara district and BHC-IV in Mbarara district in the rural setting, both about the same distance from MRRH. These facilities were selected because most births take place there owing to fully functioning theatres that support any mode of birth. Municipal HC-IV was not selected because it had been used for pre-testing the study tools.

MRRH is a referral facility for the southwestern region providing both in-patient and out-patient care. It has eight main departments: mental health, medical, surgical, pediatrics, obstetrics and gynecology, theatre, intensive care unit and outpatient departments, including maternal and child health $(\mathrm{MCH})$. The postnatal clinic has ten health workers and an average daily attendance of 30 mothers. Care includes immunization, family planning, health education, infant growth monitoring and examination of postpartum mothers.

At the time of the study, BHC-IV had eight midwives and an average daily attendance of 16 mothers and $\mathrm{KHC}$ IV had four midwives and an average of 30 mothers who attended the clinic daily, plus approximately 6 births recorded daily.

\section{Study population and period}

Data was collected from $11^{\text {th }}$ November 2019 to $10^{\text {th }}$ June 2020. The study targeted mothers who visited the postnatal clinic for their routine 6-week postpartum visit according to the Ugandan MOH Guidelines [19]. Sample size was determined using a single population formula with correction for finite population [20]. That is:

$n=N^{*} \mathrm{X} /(\mathrm{X}+\mathrm{N}-1)$, Where,

$\mathrm{X}=\mathrm{Z} \alpha / 22\urcorner^{*} p^{*}(1-p) / \mathrm{MOE} 2$,

$Z \alpha / 2$ is the critical value of the normal distribution at $\alpha / 2$ for $\alpha$ of $0.05=1.96$,

MOE is the margin of error considered at $5 \%$, 
$p=$ expected proportion of mothers with diagnosis of PPD,

$N$ is the population size of mothers who delivered at the selected study sites over the recent 3-month period.

The minimum sample size targeted was 231 mothers attending post-natal clinics in the study sites. With additional $20 \%$ to cater for attrition, the final sample size was 292 mothers.

\section{Sampling procedure}

Using proportionate stratified consecutive sampling, the sample size was divided with the health facility as a stratum. This was based on the number of women giving birth in each health facility the previous 3 months. The probability proportionate to size was used to determine the number of mothers that were enrolled at each stratum to meet the calculated sample size of 292. Mothers within each health facility were selected consecutively based on the eligibility criteria until the sample size apportioned for each stratum had been attained.

\section{Inclusion and exclusion criteria}

Women were considered eligible if they were between 18 and 49 years old and attended the clinic during the $6^{\text {th }}$ to $8^{\text {th }}$ week postpartum in the post-natal clinic. Mothers were excluded, if they reported to the clinic for illness with complaints of high-grade fever, high blood pressure, vaginal bleeding or were unable to respond to the questions. In MRRH, mothers were also excluded if they were residing outside the boundaries of Mbarara city.

\section{Study variables}

Main outcome measure was PPD among mothers. Women were classified as PPD if they had positive DSM-V criteria [21]. This was a binary variable coded 0 for PPD absent and 1 for PPD present. Independent variables were socio-demographic characteristics, maternal obstetric and medical factors, baby factors and social support factors.

\section{Measurements}

Maternal age was reported by the women at the time of data collection and grouped into four categories: younger than 20 years for adolescents, 20 to 24 years for young mothers, 25 to 34 years for middle reproductive and 35 to 49 years for older reproductive age.

Parity is the number of pregnancies from 28 weeks.

Residence was classified as either rural or urban. For this study, urban was defined as people who were residents of Mbarara city with good accessibility to health care, road and commercial settings; while rural areas have a low population density and large amounts of undeveloped land [22, 23].

Housing was classified as either temporary (grassthatched houses) or permanent (brick-built houses with iron sheets).

Obstetric complications are birth complications the mother had while giving birth, listed as excessive bleeding, obstructed labor, infection or high blood pressure.

Maternal medical history is the state of the mother at the time of birth. The factors collected were HIV positive or not and other chronic conditions like diabetes mellitus, hypertension or any others.

Occupation was referred to as how people earned a living, ranging from being unemployed to being employed as a professional or laborer according to Uganda Bureau of Statistics [24, 25].

Social support is support respondents receive from spouses, family members, and friends. This was measured by the Maternal Social Support Scale (MSSS) [26]. This validated tool measures perceived social support from family, partners, and friends. Participants are asked the extent of support from their significant others' on a 5 -point Likert scale, where 1 is never and 5 always. Scoring for two items is reversed. Scores range from 0 to 30 , categorized as low (0-18), medium (19-24) and adequate $(>24)$ [26]. Cronbach's alpha value for this tool was documented as between 0.71 and 0.90 when applied to Syrian women in Jordan [27].

Baby related factors were the fetal factors in the current delivery that were associated with PPD. Baby related factors includes the sex of the baby, complications at birth, delivered baby at 9 months, baby having health problems them, baby who was hospitalized in the last 6 weeks, baby breast feeding well and problems with baby sleeping. The factors were recorded as a Yes/No response.

Baby crying extensively is the baby crying more excessive than normal although this information was subjectively reported by the mother and recorded as a Yes/No response.

\section{Data collection tools}

This study used a Diagnostic and Statistical Manual of Mental Disorders, 5th Edition (DSM-V) to determine the prevalence of PPD. We also used a validated structured interviewer administered questionnaire to gather data about participants' characteristics to determine possible factors associated with PPD. The questionnaire had four sections with the following content: socio-demographic data, baby-related factors, maternal obstetric and medical factors and the final section contained the social support factors. These questions were adopted from earlier studies [28]. 


\section{Data collection and data quality assurance}

The English version of the questionnaire (see supplementary file) was translated to the local language (Runyankore-Rukiga) and back to English to check for consistency and accuracy. Four graduate nurses, one diploma midwife, one psychiatric clinical officer and two psychiatrists were trained as research assistants and clinicians for the three study sites. The first author and co-authors closely managed the data collection process. Training of overall content of the tools and data collection processes was conducted over five consecutive days for data collectors. We pre-tested the tools on $5 \%$ of the calculated sample size of postpartum mothers in Municipal HC-IV and revisions made as necessary to improve on contextual validity.

After consent and enrollment of eligible postpartum mothers in each site, they were first subjected to the questionnaire by nurses or midwives. All the participants were then moved to another private room where they were clinically examined by a psychiatrist using DSM-V to establish presence or absence of PPD.

\section{Data analysis}

Collected data were checked manually for completeness and consistency to minimize data entry errors. Coded data were then entered into EPI Info software version 7.2. Appropriate data validation and cleaning was done prior to exporting the database for analysis. The dataset was then imported into STATA software version 13.0 for analysis (Stata Corp, College Station, TX, USA).

Participants' characteristics were described using proportions for categorical variables. Prevalence of PPD was calculated as a proportion of postpartum mothers diagnosed with PPD using DSM-V criteria out of all mothers enrolled into the study and expressed as a percentage with corresponding 95\% confidence interval (CI). Prevalence was also expressed and compared by site, age categories and parity using Chi-square test. Statistical significance was achieved when $p \leq 0.05$.

The dependent variable was the dichotomous presence or absence of PPD. The bivariate analyses used Chisquare exact test to determine correlations between each independent variable with the dependent variable. Unadjusted odds ratios with their corresponding 95\% CI are reported.

The independent variables in bivariate analysis with $p$-values $<0.1$ were included in multivariate logistic regression analysis. Absence of multicollinearity among independent variables was confirmed [29]. A manual back-ward stepwise selection method was used in the final multivariate analysis with factors bearing independent significant associations with PPD. The goodness-of-fit test was performed on the final model to assess its quality. Factors in the final multivariate model are reported together with their adjusted odds ratios (aORs) and 95\% CI.

\section{Results}

\section{Participant characteristics}

Of the total of 580 women who were approached between November 2019 and June 2020, 275 were ineligible to participate for being less than 6 weeks and more than 8 weeks postpartum. Seventeen potential participants declined participation due to time constraints (11) and having ill children (6). There were 292 postpartum mothers who consented and enrolled into the study, with 152 enrolled from MRRH, 93 from BHC-IV and 47 from KHC-IV.

\section{Sociodemographic characteristics}

The majority of mothers were between 20 and 34 years (80.1\%), were married (95.2\%), identified as Banyankore by tribe $(76.4 \%)$, had attained at least secondary school education (36.3\%), were residing in urban areas (66.1\%) and were dwelling in permanent houses (79.5\%) (Table 1).

\section{Obstetric and medical characteristics}

The majority of participants had given birth to at least two children prior to the current pregnancy $(63.1 \%)$ and had not experienced previous child deaths (89.4\%). In the current pregnancy, most mothers (96.9\%) gave birth in health facilities with skilled health workers and by vaginal route $(80.2 \%)$. Overall, $17.5 \%$ were HIV positive and $88.7 \%$ were aware of the HIV status of their spouses (Table 1).

\section{Baby-related characteristics}

Most babies were male (51.4\%) and $10.3 \%$ had complications or abnormalities at birth. Key complications included infections (6.7\%), jaundice (30\%), birth asphyxia (23.3\%), congenital anomalies (16.7\%), and (10.3\%) preterm birth. Health problems after birth included $9.3 \%$ of babies who had been hospitalized in the prior 6 weeks, $3.8 \%$ of babies were not breastfeeding well, $33.9 \%$ had sleeping problems, $22.6 \%$ cried excessively, and $20.9 \%$ were difficult to console (Table 2).

\section{Social support among participants}

Most mothers experienced medium social support (62.3\%), 15.1\% perceived low social support from husband/partners, family, and friends and $22.6 \%$ had adequate social support. Types of support provided by spouses included helping with household chores (72.3\%), babysitting (78.1\%), financial support that paid for clinic visits (91.4\%), nutritional support (90.8\%) and counseling 
Table 1 Sociodemographic, maternal obstetric/medical and bivariate analysis of factors associated with postpartum depression

\begin{tabular}{|c|c|c|c|c|c|}
\hline Variable & $n(\%)$ & $\begin{array}{l}\text { Depressed } \\
\text { No, } n(\%)\end{array}$ & $\begin{array}{l}\text { Not Depressed } \\
\text { Yes, } n(\%)\end{array}$ & $\begin{array}{l}\text { Unadjusted } \\
\text { OR ( } 95 \% \mathrm{CI})\end{array}$ & $P$-value \\
\hline \multicolumn{6}{|l|}{ Social-Demographic Factors } \\
\hline Health facility & & & & & 0.260 \\
\hline Bwizibwera & $93(32.8)$ & $63(67.7)$ & $30(32.3)$ & 1.0 & \\
\hline Kinoni & $47(16.1)$ & $33(70.2)$ & $14(29.8)$ & $0.9(0.42-1.91)$ & \\
\hline $\mathrm{MRRH}$ & $152(52.1)$ & $117(77.0)$ & $35(23.0)$ & $0.6(0.35-1.12)$ & \\
\hline${ }^{\mathrm{C}}$ Residence & & & & & 0.005 \\
\hline Rural & $99(33.9)$ & $62(62.6)$ & $37(37.4)$ & $2.1(1.26-3.65)$ & \\
\hline Urban & $193(66.1)$ & $151(78.2)$ & $42(21.8)$ & 1.0 & \\
\hline Age (years) & & & & & 0.175 \\
\hline$<20$ & $16(5.5)$ & $8(50.0)$ & $8(50.0)$ & $3.4(1.16-10.16)$ & \\
\hline $20-24$ & $102(34.9)$ & $79(77.5)$ & $23(22.6)$ & 1.0 & \\
\hline $25-34$ & $132(45.2)$ & $95(72.0)$ & $37(28.0)$ & $1.3(0.73-2.44)$ & \\
\hline $35-49$ & $42(14.4)$ & $31(73.8)$ & $11(26.2)$ & $1.2(0.53-2.79)$ & \\
\hline Tribe & & & & & 0.155 \\
\hline Munyankore & $223(76.4)$ & $159(71.3)$ & $64(28.7)$ & 1.0 & \\
\hline Muganda & $18(6.2)$ & $11(61.1)$ & $7(38.9)$ & $1.6(0.59-4.26)$ & \\
\hline Mukiga & $31(10.6)$ & $26(83.9)$ & $5(16.1)$ & $0.5(0.18-1.30)$ & \\
\hline Others & $20(6.8)$ & $17(85.0)$ & $3(15.0)$ & $0.4(0.12-1.55)$ & \\
\hline Education & & & & & 0.281 \\
\hline None & $4(1.4)$ & $2(50.0)$ & $32(33.0)$ & 1.0 & \\
\hline Primary & $97(33.2)$ & $65(67.0)$ & $25(23.6)$ & $0.5(0.67-3.66)$ & \\
\hline Secondary & $106(36.3)$ & $81(76.4)$ & $20(23.5)$ & $0.3(0.41-2.31)$ & \\
\hline Tertiary & $85(29.1)$ & $65(76.5)$ & $79(27.1)$ & $0.3(0.41-2.33)$ & \\
\hline Marital status & & & & & 0.467 \\
\hline Unmarried & $14(4.8)$ & $9(64.3)$ & $5(35.7)$ & 1.0 & \\
\hline Married & $278(95.2)$ & $204(73.4)$ & $74(26.6)$ & $0.7(0.21-2.01)$ & \\
\hline Religion & & & & & 0.415 \\
\hline Catholic & $93(31.8)$ & $2(50.0)$ & $2(50.0)$ & 1.0 & \\
\hline Protestant & $145(49.7)$ & $65(67.0)$ & $65(67.0)$ & $0.9(0.52-1.73)$ & \\
\hline Moslem & $24(8.2)$ & $81(76.4)$ & $81(76.4)$ & $1.2(0.44-3.20)$ & \\
\hline Others & $30(10.3)$ & $65(76.5)$ & $65(76.5)$ & $1.9(0.81-4.55)$ & \\
\hline Occupation & & & & & 0.096 \\
\hline Unemployed & $60(20.5)$ & $46(76.7)$ & $14(23.3)$ & 1.0 & \\
\hline Business & $114(39.0)$ & $87(76.3)$ & $27(23.7)$ & $1.0(0.49-2.13)$ & \\
\hline Subsistence farmer & $57(19.5)$ & $35(61.4)$ & $22(38.6)$ & $2.1(0.93-4.60)$ & \\
\hline Professional & $51(17.5)$ & $40(78.4)$ & $11(21.6)$ & $0.9(0.37-2.21)$ & \\
\hline Manual labourer & $10(3.4)$ & $5(50.0)$ & $5(50.0)$ & $3.3(0.83-13.01)$ & \\
\hline Housing & & & & & 0.127 \\
\hline Temporary & $232(79.5)$ & $174(75.0)$ & $58(25.0)$ & 1.0 & \\
\hline Permanent & $60(20.5)$ & $39(65.0)$ & $21(35.0)$ & $1.6(0.88-2.97)$ & \\
\hline \multicolumn{6}{|l|}{ Maternal Medical Factors } \\
\hline 'HIV status & & & & & 0.002 \\
\hline Positive & $51(17.5)$ & $28(54.9)$ & $23(45.1)$ & $2.7(1.45-5.08)$ & \\
\hline Negative & $241(82.5)$ & $185(76.8)$ & $56(23.2)$ & 1.0 & \\
\hline${ }^{c}$ Known HIV status of husband ${ }^{b}$ & & & & & 0.042 \\
\hline No & $33(11.3)$ & 19 (57.6) & $14(42.4)$ & $2.2(1.04-4.63)$ & \\
\hline Yes & $259(88.7)$ & $194(74.9)$ & $65(25.1)$ & 1.0 & \\
\hline
\end{tabular}


Table 1 (continued)

\begin{tabular}{|c|c|c|c|c|c|}
\hline Variable & $n(\%)$ & $\begin{array}{l}\text { Depressed } \\
\text { No, } n(\%)\end{array}$ & $\begin{array}{l}\text { Not Depressed } \\
\text { Yes, } n(\%)\end{array}$ & $\begin{array}{l}\text { Unadjusted } \\
\text { OR }(95 \% \mathrm{Cl})\end{array}$ & P-value \\
\hline Chronic medical conditions & & & & & 0.193 \\
\hline No & $272(93.2)$ & $201(73.9)$ & $71(26.1)$ & 1.0 & \\
\hline Yes & $20(6.8)$ & $12(60.0)$ & $8(40.0)$ & $1.9(0.74-4.81)$ & \\
\hline Number of children & & & & & 0.380 \\
\hline 1 & $108(37.0)$ & $81(75.0)$ & $27(25.0)$ & 1.0 & \\
\hline $2-4$ & $152(52.1)$ & $112(73.7)$ & $40(26.3)$ & $1.1(0.61-1.89)$ & \\
\hline 5 or more & $32(11.0)$ & $20(62.5)$ & $12(37.5)$ & $1.8(0.77-4.16)$ & \\
\hline Deceased children & & & & & 0.407 \\
\hline None & $261(89.4)$ & $188(72.0)$ & $73(28.0)$ & 1.0 & \\
\hline Less than half & $16(5.5)$ & $12(75.0)$ & $4(25.0)$ & $0.9(0.27-2.75)$ & \\
\hline More than half & $15(5.1)$ & $13(86.7)$ & $2(13.3)$ & $0.4(0.09-1.80)$ & \\
\hline Birth place recent pregnancy ${ }^{a}$ & & & & & 0.255 \\
\hline Health facility & $283(96.9)$ & $208(73.5)$ & $75(26.5)$ & 1.0 & \\
\hline Not health facility & $9(3.1)$ & $5(55.6)$ & $4(44.4)$ & $2.2(0.58-8.48)$ & \\
\hline${ }^{c}$ Complication in recent pregnancy & & & & & 0.015 \\
\hline No & $238(81.5)$ & $181(76.1)$ & $57(24.0)$ & 1.0 & \\
\hline Yes & $54(18.5)$ & $32(59.3)$ & $22(40.7)$ & $2.2(1.18-4.05)$ & \\
\hline Mode of birth in recent pregnancy & & & & & 0.959 \\
\hline Vaginal birth & $236(80.8)$ & $172(72.9)$ & $64(27.1)$ & 1.0 & \\
\hline Caesarian section & $56(19.2)$ & $41(73.2)$ & $15(26.8)$ & $1.0(0.51-1.90)$ & \\
\hline
\end{tabular}

a Birth with a skilled health worker is not expected in home birth

${ }^{b}$ Father of the baby

${ }^{\mathrm{C}}$ Factors significant at bivariate analysis

support (83.2\%). Most mothers (71.3\%) were aware of the condition of PPD (Table 2).

\section{Prevalence of PPD}

The overall prevalence of PPD was 79/292 (27.1\%; 95\% CI 22.2-32.5). On stratification by health facility, BHC-IV had the highest prevalence of 30/93 (32.3\%; 95\% CI 23.4$42.6)$ as compared to $14 / 47$ (29.8\%; $95 \%$ CI $18.1-44.8)$ in KHC-IV and 35/152 (23\%; 95\% CI 17.0-30.5) in MRRH, however this difference in prevalence between the study sites was not statistically significant. The prevalence of PPD did not vary in a statistically significant way by maternal age, the number of previous births or mode of delivery.

\section{Factors associated with PPD}

The only sociodemographic factor with an association to PPD was place of residence. Associated maternal medical factors were HIV positive serology status of the mother, unknown HIV serology status of the husband and birth complications in recent pregnancy (Table 1). Factors related to the baby were not breast feeding well, a baby who cried excessively and perceived social factors. In total, the chi-square bivariate analysis revealed seven factors with statistically significant associations to PPD. Occupation was included in the logistic regression model because the $p$-value was less than 0.1 making a total of eight factors (Table 1 ).

\section{Multivariate regression analysis}

The overall mean variance inflation factor (VIF) for all independent variables together was 1.21 , meeting assumptions for logistic regression modeling [29]. The non-significant goodness - of - fit test based on the Hosmer and Lemeshow chi-square indicated a good fit of the model $(p=0.28)$ [30]. The classification table indicated the model improved the ability to accurately predict PPD outcome from $27 \%$ (null model without predictors) to $74 \%$ with the inclusion of the predictor variables.

In the final model, five variables demonstrated statistically significant predictive values for PPD. These were perceived social support, HIV positive serology status of the mothers, rural residence, any complication 
Table 2 Baby factors, social support characteristics and bivariate analysis of factors associated with postpartum depression

\begin{tabular}{|c|c|c|c|c|c|}
\hline Variable & $n(\%)$ & $\begin{array}{l}\text { Depressed } \\
\text { No, } n(\%)\end{array}$ & $\begin{array}{l}\text { Not Depressed } \\
\text { Yes, } n(\%)\end{array}$ & $\begin{array}{l}\text { Unadjusted } \\
\text { OR }(95 \% \mathrm{Cl})\end{array}$ & P-value \\
\hline \multicolumn{6}{|l|}{ Baby- Related Factors } \\
\hline Sex of the baby & & & & & 0.523 \\
\hline Male & $150(51.4)$ & $107(71.3)$ & $43(28.7)$ & 1.0 & \\
\hline Female & $142(48.6)$ & $106(74.7)$ & $36(25.4)$ & $0.8(0.50-1.42)$ & \\
\hline Abnormality/complications at birth & & & & & 0.103 \\
\hline No & $262(89.7)$ & $195(74.4)$ & $67(25.6)$ & 1.0 & \\
\hline Yes & $30(10.3)$ & $18(60.0)$ & $12(40.0)$ & $1.9(0.89-4.24)$ & \\
\hline Experiencing health problem now & & & & & 0.351 \\
\hline No & $253(86.6)$ & $187(73.9)$ & $66(26.1)$ & 1.0 & \\
\hline Yes & $39(13.4)$ & $26(66.7)$ & $13(33.3)$ & $1.4(0.69-2.92)$ & \\
\hline Birth at term (9 months) & & & & & 0.959 \\
\hline No & $30(10.3)$ & $22(73.3)$ & $8(26.7)$ & 1.0 & \\
\hline Yes & $262(89.7)$ & $191(73.0)$ & $71(27.1)$ & $1.0(0.44-2.40)$ & \\
\hline Baby hospitalized last 6 weeks & & & & & 0.889 \\
\hline No & $265(90.8)$ & $193(72.8)$ & $72(27.2)$ & 1.0 & \\
\hline Yes & $27(9.2)$ & $20(74.1)$ & $7(25.9)$ & $0.9(0.38-2.31)$ & \\
\hline${ }^{b}$ Baby breastfed well & & & & & 0.049 \\
\hline No & $11(3.8)$ & $5(45.5)$ & $6(54.6)$ & $3.4(1.01-11.54)$ & \\
\hline Yes & $281(96.2)$ & $208(74.0)$ & $73(26.0)$ & 1.0 & \\
\hline Problems with baby sleeping & & & & & 0.150 \\
\hline No & $193(66.1)$ & $146(75.7)$ & $47(24.4)$ & 1.0 & \\
\hline Yes & 99 (33.9) & $67(67.7)$ & $32(32.3)$ & $1.5(0.87-2.53)$ & \\
\hline baby crying excessively & & & & & 0.012 \\
\hline No & $226(77.4)$ & $173(76.6)$ & $53(23.5)$ & 1.0 & \\
\hline Yes & $66(22.6)$ & $40(60.6)$ & $26(39.4)$ & $2.1(1.19-3.80)$ & \\
\hline Baby difficult to console & & & & & 0.423 \\
\hline No & $231(79.1)$ & $171(74.0)$ & $60(26.0)$ & 1.0 & \\
\hline Yes & $61(20.9)$ & $42(68.9)$ & $19(31.2)$ & $1.3(0.70-2.39)$ & \\
\hline \multicolumn{6}{|l|}{ Social Support Factors } \\
\hline${ }^{b}$ Perceived social support & & a & & & 0.016 \\
\hline Low & $44(15.1)$ & $25(56.8)$ & $19(43.2)$ & $3.4(1.44-8.12)$ & \\
\hline Medium & $182(62.3)$ & $134(73.6)$ & $48(26.4)$ & $1.6(0.79-3.27)$ & \\
\hline Adequate & $66(22.6)$ & $54(81.8)$ & $12(18.2)$ & 1.0 & \\
\hline
\end{tabular}

${ }^{a}$ Includes support from friends, family and husband and scored as Low (0-18), Medium (19-24), Adequate (>24)

${ }^{\mathrm{b}}$ Factors significant at multivariate analysis

in recent pregnancy and a baby who cried excessively (Table 3).

\section{Discussion}

\section{Prevalence of PPD}

The prevalence of postpartum depression among the 292 mothers was $27.1 \%$, much higher than $6.1 \%$ and lower than $43 \%$, reported by previous Ugandan studies [16, 17]. It was also higher than the prevalence reported in other African countries such as Ethiopia (20.9-22.9\%), Sudan
(9.2\%), Kenya (13-18.7\%), Tanzania (12\%) and Ghana (7\%) [15].

The prevalence is, however, lower than the range of 31.7 to $50.3 \%$ reported from South Africa and 33\% in Zimbabwe [31]. The variance between our study and the other studies can be attributed to the variations in sample sizes, tools used to measure PPD, using a screening tool as a diagnostic tool without validating the tool, timing of postpartum period and socio-cultural differences. For instance, in a peri-urban health centre in Kampala women were assessed at 6 weeks 
Table 3 Results of multivariate analysis for factors independently associated with PPD

\begin{tabular}{|c|c|}
\hline Variable & Adjusted OR $(95 \% \mathrm{Cl})$ \\
\hline \multicolumn{2}{|l|}{${ }^{\text {aResidence }}$} \\
\hline Rural & $1.9(1.03-3.64)$ \\
\hline Urban & 1.0 \\
\hline \multicolumn{2}{|l|}{ Occupation } \\
\hline Unemployed & 1.0 \\
\hline Business & $1.1(0.89-2.36)$ \\
\hline Peasant farmer & $1.4(0.57-3.48)$ \\
\hline Professional & $1.3(0.49-3.42)$ \\
\hline Manual labourer & $2.3(0.51-10.62)$ \\
\hline \multicolumn{2}{|c|}{ Baby breastfeeding well } \\
\hline No & $3.4(0.94-12.15)$ \\
\hline Yes & 1.0 \\
\hline \multicolumn{2}{|c|}{ aBaby cries excessively } \\
\hline No & 1.0 \\
\hline Yes & $2.2(1.15-4.08)$ \\
\hline \multicolumn{2}{|l|}{ aHIV status } \\
\hline Positive & $2.1(1.04-4.17)$ \\
\hline Negative & 1.0 \\
\hline \multicolumn{2}{|c|}{ Known HIV status of husband } \\
\hline No & 1.0 \\
\hline Yes & $1.7(0.71-3.85)$ \\
\hline \multicolumn{2}{|c|}{${ }^{\mathrm{a} A n y}$ complication in recent pregnancy } \\
\hline No & 1.0 \\
\hline Yes & $2.0(1.00-3.85)$ \\
\hline \multicolumn{2}{|c|}{ aPerceived social support } \\
\hline Low & $2.3(0.87-6.17)$ \\
\hline Medium & $1.3(0.62-2.85)$ \\
\hline Adequate & 1.0 \\
\hline
\end{tabular}

${ }^{a}$ Model characteristics: $N=292 ; \chi^{2}(11)=38.54, p<.001$, Pseudo- $R^{2}: .1130$

postpartum and prevalence of PPD was determined using a 25-Item Self-Reporting Questionnaire (SRQ25) and MINI to confirm major depression [16]. MINI, however, was more inferior than DSM-Vin diagnosing for PPD since it broadly assessed for major depression and this study was implemented 15 years ago. Other reports of much higher prevalence in southwestern Uganda may be attributed to use of the untranslated EPDS screening tool used with mothers who may have limited understanding of English [17]. Further, the EPDS was administered as a diagnostic tool instead of screening, for which it was originally designed. Use as a diagnostic tool without adaption to the local context, is likely to over-estimate or under-estimate the burden and prevalence of PPD. In our study, the prevalence was determined by clinically diagnosing mothers with DSM-V, which is the "gold standard" diagnostic criterion. These diagnostic tools were translated to the local language, Runyankore, for participants with limited English proficiency.

\section{Factors associated with PPD}

Mothers' perceived social support by husbands/partners, family and friends; HIV positive status of the women; babies who cried excessively; women with complications in the current pregnancy; and rural residence were significantly associated with a clinical diagnosis of PPD. Social support is important during and after childbirth which is in agreement with studies indicating social support as a protective factor among women with PPD [32]. In rural South Africa, a rate of $60.9 \%$ of mothers without family or social support suffered from severe depression as compared to $28.2 \%$ who had moderate depression [32]. Several studies from Cameroon, Ethiopia and Pakistan identified lack of social support as a risk factor for PPD [33-36].

During the post-partum period women should be recovering from the stress of pregnancy, birth and physiological adaptation after childbirth. To reduce this postpartum related stress, mothers are advised to receive social support from their husbands/partners, family relations and friends. Social support can prevent PPD and its after effects, optimizing mothers' positive self-images and enhancing quality of life [37].

There is scanty literature reporting the association between new-borns who cry excessively and PPD. Participants who reported their babies cried excessively in present study were 2.2 times more likely to experience PPD. If babies cry much and women receive no or low social support from husbands/partners, they may be deprived of sleep leading to PPD [38]. Babies who cry excessively may not be breastfed well and this causes anxiety in the mothers, hence potentially contributing to PPD development $[39,40]$. PPD adversely influences infant development and behaviour such as crying and breast feeding, but it remains unclear if this correlation is causal and if so, in what direction [41]. Other behaviour influenced by PPD includes irritability and difficulty in soothing and consoling the baby.

HIV-positive participants in the present study were 2.1 times more likely to experience PPD than HIV-negative ones. Other studies in similar settings have reported an increased risk of PPD among HIV-infected mothers, consistent with our study [42-44]. Women living with HIV/ AIDS are at high risk of PPD, because they face several risk factors like lack of social support, stigma and discrimination [45].

The study setting may have an effect on the correlation between HIV status and PPD. Research from high income countries predominantly report no association between HIV and PPD prevalence [46-48]. This lack 
of association could be explained by the type of care provided to postpartum mothers following childbirth. Mothers in high income countries may be more likely to receive systematic follow up and counseling from nurses and social workers in accordance with local government health systems than mothers in low-resourced settings. The children born to mothers in high income countries are less likely to be HIV positive because of the structured Prevention of Mother To Child Transmission (PMTCT) programs which reduce transmission and incidence of newborn babies with HIV [49]. A significant risk for PPD was, however, reported in HIV-positive mothers whose infants were also diagnosed with HIV [50]. This calls for integration of psychosocial support in the postpartum care given to HIV positive women.

Mothers with a history of any birth complications in the most recent pregnancy were twice more likely to suffer from PPD as compared to those without complications. Each year in Africa, 30 million women become pregnant and about 250,000 of them die from pregnancyrelated causes. Several studies identified a strong association between obstetric complications and PPD [51-54]. A study delineating the association between heavy postpartum haemorrhage and PPD in Uppsala, Sweden observed direct and positive influences between anaemia at discharge from hospital and self-reported bad birth experiences with PPD [52]. Anaemia is a consequence of excessive vaginal bleeding or postpartum haemorrhage and mothers are likely to present with fatigue, reduced cognitive abilities, emotional disturbances and consequently PPD [55]. When these mothers are distressed, they are likely not breastfeeding well, hence their babies may be more apt to cry excessively.

Pre-eclampsia has also been shown as a risk factor to PPD [56]. Serotonin levels in blood are known to be high in women suffering from hypertension [57]. This may lead to decreased serotonin levels in the mothers' brain, thereby causing PPD. Women who experience birth complications are likely to experience physical and psychological morbidity after birth [58].

Participants in the present study who live in rural areas, were twice more likely to experience PPD compared to those living in urban areas. The impact of place of residence has not been adequately studied on risk of suffering from PPD in low-resourced settings. However, studies from middle income countries, such as Bangladesh, India, Iran and Pakistan concur with our findings that PPD is more prevalent in mothers residing in rural settings [59-63]. Rural Ugandan women may be more at risk of suffering from PPD because they mostly depend on subsistence agriculture with low standards of living and are commonly poorer than women in urban settings $[22,25]$.
One study reported no association between residing in urban or rural areas and PPD [64]. Conversely in highincome countries like Canada and Australia higher rates of PPD are reported among mothers in urban settings than those in rural areas [65-67]. This is possibly due to geographical differences between urban settings in highincome and low-income countries. More frequently in high-income countries, urban settings may be associated with dense populations, higher rates of interpersonal violence, poorer health and lower social support as compared to rural settings [65].

\section{Strengths and limitations}

A key strength was the use of a "gold standard" DSM-V to clinically diagnose and confirm mothers suffering from PPD.

This study was limited to women having given birth in public health facilities, hence results should cautiously be generalized to private health facilities, given the expected differences in socio-demographic and economic characteristics of patients that visit these two settings. Previous pregnancy experience was not the focus of this study whereas some literature highlights a strong association between previous birth experience and pregnancy related variables. In addition, the cross-sectional design limits the capacity to draw any causal implications especially between some variables and PPD, given its limitation in establishing temporal relationships between independent variables and outcome [68]. The fact that two-thirds of the participating mothers were from urban settings with good access to health facilities could have resulted in an underestimate of prevalence of PPD. This study was carried out during the global pandemic of COVID-19 that may have increased PPD prevalence in Uganda. Despite these limitations, this is the first study in the Uganda setting that clinically diagnosed mothers with PPD in rural and urban public health facilities. Our study findings are useful to inform the magnitude and risk factors associated with PPD in Uganda for planning and integrating PPD prevention and care to women at risk.

\section{Implications and recommendations}

These findings can be useful for practice and policy in Uganda and other low-resource settings. They point out the need to increase public awareness about PPD and its associated factors. The high prevalence of PPD among Ugandan women indicates an urgent need to identify pregnant women at increased threat of PPD to mitigate the risk or implement therapies to manage the condition. Because of the high prevalence, PPD screening and intervention need to be integrated with maternal health care in public health facilities, especially for mothers at risk of 
PPD. In addition, PPD burden needs to be evaluated in mothers attending private health facilities so as to guide the scaling of appropriate interventions to these facilities. Midwives who attend to these mothers should be trained to screen for PPD.

Such training would require development of educational initiatives to disseminate the risks for PPD and adverse consequences of unidentified and untreated PPD. There is a need to validate a culturally and linguistically relevant screening tool that can be applied by all nurses and midwives for PPD. Validation of the EPDS screening tool through translation and establishment of culturally appropriate cut-off point for optimal sensitivity and specificity has been implemented in another arm of this current study. Results of this validation will be reported elsewhere. Inclusion of such a validated screening tool within the Uganda Clinical Guidelines will empower Ugandan midwives to practice at their highest potential to mitigate the prevalence and consequences of PPD.

Women who are HIV infected, reside in rural settings, whose babies cry excessively, have low social support and who have birth complications may be a particularly important focus for Ugandan intervention strategies to prevent and reduce PPD. Mothers' perceptions of adequate social support and their relationships with their relatives and friends are potential facilitators to reduce burden of PPD. This strength should be considered in development of community interventions to benefit pregnant women, focusing on improving family and community relationships.

These findings are indicative of the need for midwives and nurses working in rural areas to ensure comprehensive assessment of postpartum mothers, most especially those living with HIV to offer the best chance of implementing holistic care for postpartum women suffering from PPD.

Future studies may utilize longitudinal methods to track a single group of mothers to examine causal relationships between depression and risk factors identified in this study. Although various socio-demographic, maternal obstetric medical, baby related and social support correlates were examined in this study, prior research has shown that biological factors such as oxytocin levels and personality traits are also significantly correlated with PPD. More personal and biological predictors could be examined in future research.

\section{Conclusion}

More than one-quarter of the women in this study demonstrated clinical diagnosis of PPD, indicating a high prevalence in south-western Uganda. Whereas the most recent HIV-prevalence in south-western Uganda stands at $7.9 \%$, comparable to $8 \%$ in the whole of Uganda, PPD is more prevalent among HIV-positive mothers and residing in rural settings [25]. Addressing PPD in this context, nurses in postnatal clinics should tailor interventions and target HIV-positive mothers, those with low social support or a history of birth complications, having babies that cry excessively, or reside in rural settings. Nursemidwives in maternal health should be aware of PPD and be able to provide immediate holistic care.

This study provides an important contribution to the fight against maternal mental illness and its associated factors. A qualitative study to understand the lived experiences, challenges and coping mechanisms can be done in this health district.

\section{Abbreviations}

BHC-IV: Bwizibwera Health Centre IV; Cl: Confidence interval; DSM-V: Diagnostic and Statistical Manual of Mental Disorders, 5th Edition; EPDS: Edinburgh Postnatal Depression Scale; HCW: Health care workers; KHC-IV: Kinoni Health Centre IV; PPD: Postpartum depression; SRQ: Self-Reporting Questionnaire (SRQ-20); SDG: Sustainable Development Goals; HIV: Human Immunodeficiency Virus; MCH: Maternal and child health; MRRH: Mbarara Regional Referral Hospital; MSSS: Maternal Social Support Scale.

\section{Supplementary Information}

The online version contains supplementary material available at https://doi. org/10.1186/s12884-021-03967-3.

Additional file 1.

\section{Acknowledgements}

We are grateful to all mothers who participated in this research.

\section{Authors' contributions}

CA conceived and designed the study. CA and DA implemented the study and conducted data analysis. GZR, GN and LB supervised the study. CA, GZR, GN, NJ, DA, SNC and LB interpreted study results: CA wrote the first draft of the manuscript. CA, GZR, GN, NJ, DA, SNC and LB reviewed and corrected the draft manuscript. All authors read and approved the final manuscript.

\section{Funding}

No funding for this study.

\section{Availability of data and materials}

The data that support the findings of this study are available from the first and corresponding author, Catherine Atuhaire but restrictions apply under license for the current study.

\section{Declarations}

\section{Ethics approval and consent to participate}

Ethical approval was obtained from the Mbarara University of Science and Technology (MUST), Faculty Research and Ethics Committee (FREC) and Institutional Review Committee (MUST-IRB). After obtaining ethical approval, permission was sought from the hospital Director, Mbarara Regional Referral Hospital and the District Health Officers of Bwizibwera and Kinoni HCIV. The approval letters were then presented to Uganda National Council of Science and Technology (UNCST) for approval and further submitted to the head of department obstetrics and gynecology ward, MRRH, heads of departments, postnatal wards of Bwizibwera and Kinoni health center IVs, plus the Local Councils in Rwampara and Kashari County, Mbarara District.The study further adhered to the following key ethical principles: informed consent, confidentiality, and anonymity, voluntary participation, protection of participants 
from harm, trustworthiness, and transparency. Written informed consent was sought from all the participants and were identified by number and not their names. The findings from this study were stored in a computer secured with a password for purposes of confidentiality. For those mothers who were diagnosed with PPD, they were referred to the Mental Health Clinic for further clinical assessment and management. While administering the EPDS, DSM-V and the questionnaire, participants had immediate access to counseling services in the event that they triggered emotions that posed the participants' health and wellbeing.

\section{Consent for publication}

Not applicable.

\section{Competing interests}

The authors declare no competing interest.

Received: 23 November 2020 Accepted: 25 June 2021

Published online: 12 July 2021

\section{References}

1. Cantwell R, Clutton-Brock T, Cooper G, et al. Saving mothers'lives: reviewing maternal deaths to make motherhood safer: 2006-2008. The eighth report of the confidential enquiries into maternal deaths in the United Kingdom. BJOG. 2011;118:1.

2. Oates M. Perinatal psychiatric disorders: a leading cause of maternal morbidity and mortality. Br Med Bull. 2003;67(1):219-29.

3. Miranda JJ, Patel V. Achieving the millennium development goals: does mental health play a role? PLoS Med. 2005;2(10):e291

4. Asaye MM, Muche HA, Zelalem ED. Prevalence and predictors of postpartum depression: Northwest Ethiopia. Psychiatry J. 2020;2020:9565678.

5. Babatunde T, Moreno-Leguizamon CJ. Daily and cultural issues of postnatal depression in African women immigrants in South East London: tips for health professionals. Nurs Res Pract. 2012:2012:181640.

6. Pearlstein T, Howard M, Salisbury A, Zlotnick C. Postpartum depression. Am J Obstet Gynecol. 2009;200(4):357-64.

7. Cox J, Holden J, Sagovsky R. Edinburgh postnatal depression scale (EPDS). Br J Psychiatry. 1987;150:782-6.

8. Yonemoto N, Dowswell T, Nagai S, Mori R. Schedules for home visits in the early postpartum period. Evid Based Child Health. 2014;9(1):5-99.

9. Sulyman D, Ayanda KA, Dattijo LM, Aminu BM. Postnatal depression and its associated factors among Northeastern Nigerian women. Ann Trop Med PH. 2016:9(3):184.

10. Werner E, Miller M, Osborne LM, Kuzava S, Monk C. Preventing postpartum depression: review and recommendations. Arch Womens Ment Health. 2015;18(1):41-60.

11. Bährer-Kohler S. Introduction to the book: Global mental health: promotion and prevention. Global Mental Health. Cham: Springer; 2017. p. 1-8.

12. Fisher J, Mello MCd, Patel V, et al. Prevalence and determinants of common perinatal mental disorders in women in low-and lowermiddle-income countries: a systematic review. Bull World Health Organ. 2012;90:139-49.

13. Gelaye B, Rondon MB, Araya R, Williams MA. Epidemiology of maternal depression, risk factors, and child outcomes in low-income and middleincome countries. Lancet Psychiatry. 2016;3(10):973-82.

14. Norhayati M, Hazlina NN, Asrenee A, Emilin WW. Magnitude and risk factors for postpartum symptoms: a literature review. J Affect Disord. 2015;175:34-52.

15. Atuhaire C, Brennaman L, Cumber SN, Rukundo GZ, Nambozi G. The magnitude of postpartum depression among mothers in Africa: a literature review. PAMJ. 2020;37:89.

16. Nakku J, Nakasi G, Mirembe F. Postpartum major depression at six weeks in primary health care: prevalence and associated factors. Afr Health Sci. 2006;6(4):207-14.

17. Kakyo TA, Muliira JK, Mbalinda SN, Kizza IB, Muliira RS. Factors associated with depressive symptoms among postpartum mothers in a rural district in Uganda. Midwifery. 2012;28(3):374-9.

18. Halbreich U, Karkun S. Cross-cultural and social diversity of prevalence of postpartum depression and depressive symptoms. J Affect Disord. 2006;91(2-3):97-111.
19. Republic of Uganda MoH. Uganda clinical guidelines 2012: national guidelines for management of common conditions. Uganda: Ministry of Health Kampala; 2012.

20. Daniel WW. Determination of sample size for estimating proportions. Biostatistics. 1999;8:189-90.

21. Association AP. Diagnostic and statistical manual of mental disorders $\left(\right.$ DSM- $\left.5^{\circledR}\right)$. Virginia: American Psychiatric Pub; 2013.

22. Mukwaya P, Bamutaze Y, Mugarura S, Benson T. Rural-urban transformation in Uganda. J Afr Dev. 2012;14(2):169-94.

23. Statistics UBO. The national population and housing census 2014-main report. Kampala: Uganda Bureau of Statistics; 2016.

24. Commission NP. National development plan vision 2030. 2013.

25. Uganda Bureau of Statistics I. Uganda demographic and health survey 2016: key indicators report. Rockville: UBOS; 2017.

26. Webster J, Linnane JW, Dibley LM, Hinson JK, Starrenburg SE, Roberts JA. Measuring social support in pregnancy: can it be simple and meaningful? Birth. 2000;27(2):97-101.

27. Mohammad Kl, Abu Awad D, Creedy DK, Gamble J. Postpartum depression symptoms among Syrian refugee women living in Jordan. Res Nurs Health. 2018:41(6):519-24.

28. Webster J, Nicholas C, Velacott C, Cridland N, Fawcett L. Quality of life and depression following childbirth: impact of social support. Midwifery. 2011;27(5):745-9.

29. Midi H, Sarkar SK, Rana S. Collinearity diagnostics of binary logistic regression model. J Interdiscip Math. 2010;13(3):253-67.

30. Long JS, Freese J. Regression models for categorical dependent variables using Stata. Texas: Stata press; 2006.

31. Chibanda D, Mangezi W, Tshimanga M, et al. Validation of the Edinburgh Postnatal Depression Scale among women in a high HIV prevalence area in urban Zimbabwe. Arch Womens Ment Health. 2010;13(3):201-6.

32. Stellenberg EL, Abrahams JM. Prevalence of and factors influencing postnatal depression in a rural community in South Africa. Afr J Prim Health Care Fam Med. 2015;7(1):1-8.

33. e Couto TC, Brancaglion MYM, Alvim-Soares A, et al. Postpartum depression: a systematic review of the genetics involved. World J Psychiatry. 2015;5(1):103.

34. Wubetu AD, Engidaw NA, Gizachew KD. Prevalence of postpartum depression and associated factors among postnatal care attendees in Debre Berhan, Ethiopia, 2018. BMC Pregnancy Childbirth. 2020;20:1-9.

35. Engidaw NA, Gizachew KD. Prevalence of postpartum depression and associated factors among postnatal care attendees in Debre Berhan, Ethiopia, 2018. 2020.

36. Klainin P, Arthur DG. Postpartum depression in Asian cultures: a literature review. Int J Nurs Stud. 2009;46(10):1355-73.

37. Harandi TF, Taghinasab MM, Nayeri TD. The correlation of social support with mental health: a meta-analysis. Electron Physician. 2017;9(9):5212.

38. Kim TH, Connolly JA, Tamim H. The effect of social support around pregnancy on postpartum depression among Canadian teen mothers and adult mothers in the maternity experiences survey. BMC Pregnancy Childbirth. 2014;14(1):162.

39. Leung S, Arthur DG, Martinson I. Stress in women with postpartum depression: a phenomenological study. J Adv Nurs. 2005;51(4):353-60.

40. Ugarriza DN. Postpartum depressed women's explanation of depression. J Nurs Scholarsh. 2002;34(3):227-33.

41. Pawluski JL, Brain U, Hammond GL, Oberlander TF. Selective serotonin reuptake inhibitor effects on neural biomarkers of perinatal depression. Arch Womens Ment Health. 2019:22(3):431-5.

42. Kaida A, Matthews LT, Ashaba S, et al. Depression during pregnancy and the postpartum among HIV-infected women on antiretroviral therapy in Uganda. J Acquir Immune Defic Syndr (1999). 2014;67(Suppl 4):S179.

43. Antelman $G$, Kaaya $S$, Wei R, et al. Depressive symptoms increase risk of HIV disease progression and mortality among women in Tanzania. J Acquir Immune Defic Syndr (1999). 2007;44(4):470.

44. Chibanda D, Mangezi W, Tshimanga M, et al. Postnatal depression by HIV status among women in Zimbabwe. J Womens Health. 2010;19(11):2071-7.

45. Bennetts A, Shaffer N, Manopaiboon C, et al. Determinants of depression and HIV-related worry among HIV-positive women who have recently given birth, Bangkok, Thailand. Soc Sci Med. 1999;49(6):737-49. 
46. Collins CH, Zimmerman C, Howard LM. Refugee, asylum seeker, immigrant women and postnatal depression: rates and risk factors. Arch Womens Ment Health. 2011;14(1):3-11.

47. Rubin LH, Cook JA, Grey DD, et al. Perinatal depressive symptoms in HIV-infected versus HIV-uninfected women: a prospective study from preconception to postpartum. J Womens Health. 2011;20(9):1287-95.

48. Mokhele I, Nattey C, Jinga N, Mongwenyana C, Fox MP, Onoya D. Prevalence and predictors of postpartum depression by HIV status and timing of HIV diagnosis in Gauteng, South Africa. PLoS One. 2019:14(4):e0214849.

49. Coleman J, Bohlin KC, Thorson A, et al. Effectiveness of an SMS-based maternal mHealth intervention to improve clinical outcomes of HIVpositive pregnant women. AIDS Care. 2017;29(7):890-7.

50. Dow A, Dube Q, Pence BW, Van Rie A. Postpartum depression and HIV infection among women in Malawi. J Acquir Immune Defic Syndr (1999). 2014;65(3):359.

51. Adewuya AO, Fatoye FO, Ola BA, ljaodola OR, Ibigbami S-MO. Sociodemographic and obstetric risk factors for postpartum depressive symptoms in Nigerian women. J Psychiatr Pract. 2005;11(5):353-8.

52. Eckerdal P, Kollia N, Löfblad J, et al. Delineating the association between heavy postpartum haemorrhage and postpartum depression. PLoS One. 2016;11(1):e0144274.

53. Cirik DA, Yerebasmaz N, Kotan VO, et al. The impact of prenatal psychologic and obstetric parameters on postpartum depression in late-term pregnancies: a preliminary study. Taiwan J Obstet Gynecol. 2016;55(3):374-8

54. Byatt N, Hicks-Courant K, Davidson A, et al. Depression and anxiety among high-risk obstetric inpatients. Gen Hosp Psychiatry. 2014;36(6):644-9.

55. Gausia K, Ryder D, Ali M, Fisher C, Moran A, Koblinsky M. Obstetric complications and psychological well-being: experiences of Bangladeshi women during pregnancy and childbirth. J Health Popul Nutr. 2012;30(2):172.

56. Blom $E$, Jansen $P$, Verhulst $F$, et al. Perinatal complications increase the risk of postpartum depression. The Generation R Study. BJOG. 2010;117(11):1390-8.

57. Bolte AC, van Geijn HP, Dekker GA. Pathophysiology of preeclampsia and the role of serotonin. Eur J Obstet Gynecol Reprod Biol. 2001;95(1):12-21.
58. Borders N. After the afterbirth: a critical review of postpartum health relative to method of delivery. J Midwifery Womens Health. 2006;51(4):242-8.

59. Villegas L, McKay K, Dennis CL, Ross LE. Postpartum depression among rural women from developed and developing countries: a systematic review. J Rural Health. 2011;27(3):278-88.

60. Gausia K, Fisher C, Ali M, Oosthuizen J. Magnitude and contributory factors of postnatal depression: a community-based cohort study from a rural subdistrict of Bangladesh. Psychol Med. 2009;39(6):999.

61. Savarimuthu R, Ezhilarasu P, Charles H, Antonisamy B, Kurian S, Jacob K. Post-partum depression in the community: a qualitative study from rural South India. Int J Soc Psychiatry. 2010;56(1):94-102.

62. Goker A, Yanikkerem E, Demet MM, Dikayak S, Yildirim Y, Koyuncu FM. Postpartum depression: is mode of delivery a risk factor? ISRN Obstet Gynecol. 2012;2012:616759.

63. Rahman A, labal Z, Harrington R. Life events, social support and depression in childbirth: perspectives from a rural community in the developing world. Psychol Med. 2003;33(7):1161.

64. Milgrom J, Gemmill AW, Bilszta JL, et al. Antenatal risk factors for postnatal depression: a large prospective study. J Affect Disord. 2008:108(1-2):147-57.

65. Vigod SN, Tarasoff LA, Bryja B, Dennis C-L, Yudin MH, Ross LE. Relation between place of residence and postpartum depression. CMAJ. 2013;185(13):1129-35.

66. Zarghami M, Abdollahi F, Lye M-S. A comparison of the prevalence and related risk factors for post-partum depression in urban and rural areas. Iran J Psychiatry Behav Sci. 2019;13(2):62558.

67. Azad R, Fahmi R, Shrestha S, et al. Prevalence and risk factors of postpartum depression within one year after birth in urban slums of Dhaka, Bangladesh. PLoS One. 2019;14(5):e0215735.

68. Solem RC. Limitation of a cross-sectional study. Am J Orthod Dentofac Orthop. 2015;148(2):205.

\section{Publisher's Note}

Springer Nature remains neutral with regard to jurisdictional claims in published maps and institutional affiliations.
Ready to submit your research? Choose BMC and benefit from:

- fast, convenient online submission

- thorough peer review by experienced researchers in your field

- rapid publication on acceptance

- support for research data, including large and complex data types

- gold Open Access which fosters wider collaboration and increased citations

- maximum visibility for your research: over $100 \mathrm{M}$ website views per year

At $\mathrm{BMC}$, research is always in progress.

Learn more biomedcentral.com/submissions 